\title{
Arteterapia y síndrome de Asperger: ¿Por qué y para qué?
}

\section{Art-therapy and Asperger Syndrome: ¿why, and what for?}

D. Pedro José Regis Sansalonis
PhD. del Departamento de Didáctica de la Expresión Musical, Plástica y Corporal. Universidad de Jaén, Jaén, España.pregis@ujaen.es

Cómo citar: Regis,P.J (2016) Arteterapia y síndrome de Asperger:¿Por qué y para qué? Sophia 12 (2): 187-194.

\section{Resumen}

El presente artículo tiene la intención de clarificar el por qué y para qué es importante utilizar el arteterapia con el colectivo de síndrome de Asperger. Lo anterior, mediante un ejercicio de revisión bibliográfica, sobre todo en fuentes secundarias como trabajo de investigación, finalmente se reconoce la escasa bibliografía encontrada y la necesidad de seguir investigando el arteterapia en este colectivo social aún desconocido por la mayor parte de la sociedad.

Palabras clave: Arteterapia, capacidad, colectivo, educación, síndrome de Asperger.

\section{Abstract}

The purpose of this article is to explain the reason and importance of using art-therapy in groups dealing with Asperger Syndrome, through a bibliographic review, specially of secondary sources as a research work. Finally, it is recognized the scarce bibliography found, and the need of continuing to investigate art-therapy in this social group, still unknown by most of the society.

Keywords: Asperger's syndrome, art therapy, colective, education, skills and abilities. 


\section{Arteterapia: ¿Por qué su utilización?}

La inclusión en la utilización de la arteterapia en el colectivo con síndrome de Asperger, resulta interesante desde una perspectiva tanto social, educativa como terapéutica. Aquellos fines que expresa esta disciplina, ejemplifican de una forma vacua y veraz los objetivos que se pretenden en la investigación.

Como bien expresa Klein, el arteterapia:

Es el encuentro entre dos proyectos, uno artístico y el otro terapéutico. Se trata de un acompañamiento a personas con dificultades sociales, psicológicas, físicas, educativas, existenciales, a través de sus producciones artísticas (...) de tal manera que el trabajo realizado a partir de sus creaciones genere un proceso de transformación de sí misma y le ayude a integrarse en sus grupos de referencia social, de una manera crítica y creativa.(Klein, 2008:9).

El arteterapia ofrece multitud de posibilidades en el grupo social de asperger, permitiéndose descubrir una capacidad intrínseca de sus pensamientos transformando el producto que realiza y, que además, se encuentra conectado con su interior, es decir, con su personalidad. El individuo conlleva en sí un proyecto de persona, de cambio, que ocasiona un recorrido donde el sujeto descubrirá nuevos aspectos y utilidades del arte como herramienta audiovisual, creativa, socio-educativa y terapéutica, según Eisner:

Las artes nos invitan a prestar atención a las cualidades de lo que oímos, vemos, saboreamos y palpamos para poderlo experimentar; lo que buscamos en las artes es la capacidad de percibir cosas, no solo el hecho de reconocerlas. Se nos da licencia para lentificar la percepción, para examinar atentamente, para saborear las cualidades que en condiciones normales intentamos abordar con tanta eficacia que apenas notamos que están ahí. (Eisner, 2004: 22).

No se pretende que el arteterapia se limite solamente a ejercer una catarsis puntual en la actividad diaria del individuo, sino que le ayude en sus actividades cotidianas para afrontar y confrontar los problemas que van surgiendo.
Aunque para ello, sujeto y objeto deben de relacionarse entre sí, como respuesta a un desafío personal o profesional en concreto. Un ejemplo de ello puede ser el identificar cuándo otra persona nos está hablando irónicamente o está comentando frases hechas. El saber detectar estas situaciones requiere de un trabajo previo que debe de ser trabajado con el arte y sus diferentes técnicas.

Según Rosal $(1996,2001)$ la utilización guiada de las imágenes sobre todo, utilizada en población adulta y en niños con problemas de conducta, se plantea como recurso para disminuir el estrés e incrementar la seguridad y el control de la persona afectada. Gracias a técnicas como el collage o el fotomontaje, junto a la integración de diversos materiales como lápices de cera, rotuladores, ceras... resultan ser un revulsivo para conseguir lo expuesto.

Varios estudios afirman que el arteterapia presenta beneficios en personas con síndrome de Asperger, capacitándolas para un mejor desarrollo en cuanto a sus habilidades sociales y comunicativas, el poder y saber desplegar situaciones personales entre sus iguales y así, desarrollar circunstancias de interacción social, además de decrecer ciertos niveles de estrés y ansiedad (según sea la particularidad del sujeto) que pueden encontrarse en las rutinas diarias (Emery, 2004; Elkis-Albuhoff, 2008; Epp, 2008).

El arteterapia tiene el potencial de mejorar la comunicación, la expresión y la construcción de las relaciones personales cuando es aplicada en adolescentes con síndrome de Asperger. Existen modelos de terapia artística dirigidos al Trastorno del Espectro Autista (TEA) (Gabriels, 2000; Evans y Dubowski, 2001).

La terapia artística ofrece un modo de solucionar problemas visualmente. Esto fuerza a ser menos literales y reforzar la autoexpresión, y ofrece un modo no amenazante de tratar con el rechazo. Ello sustituye la necesidad de rabietas o interpretando comportamientos ofreciéndose como un medio aceptable de descarga emocional y permite al niño calmarse (Henley, 2000).

Ante una inexistencia intransigente en seguir rutinas habituales, las terapias creativas son una forma multidireccional en la relación terapéutica que 
posibilita la expresión interna de una persona a la que también debemos añadir la posibilidad de un redescubrimiento en las relaciones interpersonales (Duarte \& Simoes 2011).

El arte anima a las personas a representar su realidad y sus experiencias a través de estos medios. Las formas se representan en objetos y el acto mismo del dibujo, por ejemplo, les anima a una intención de relacionarse con el mundo que les rodea, es decir, con los demás.

Los adolescentes con síndrome de Asperger, tienden a carecer de la necesidad de relacionarse, aunque no todos. Sin embargo, la terapia artística puede servir como un camino hacia la conciencia aumentada del yo. Pienso, luego existo, como diría Sócrates. El poder experimentar con el yo, es un proceso de desarrollo. Puede ser muy difícil para algunos niños y es en particular factible o, quizás fácil, para otros.

Deben aprender a experimentar el acto de dibujar, pintar, modelar, cortar, pegar, expresar, etc. El arte siempre demostrará ser un proceso evolutivo que conduce a la siguiente etapa. La terapia de arte para niños y/o adolescentes con síndrome de Asperger, puede ser una intervención importante en base a su actividad para animar su crecimiento no solo personal sino también profesional (Emery, 2004).

A pesar de los desafíos que pueden resultar en los adolescentes con síndrome de Asperger, y ante la creación emocional y cognitiva de imágenes representativas a través de la utilización de instrumentos audiovisuales (como es en nuestro caso, la fotografía y el vídeo), estos medios son para ellos un claro ejemplo de poder obtener aquellos objetivos socio-educativos que sean eficaces como solución a aquellos problemas que se enfrentan en su entorno.

\section{¿Para qué su utilización?}

Estos adolescentes tienen déficits comunes en las áreas de socialización, falta de flexibilidad cognitiva, intereses monotemáticos, funciones ejecutivas (como por ejemplo, organizarse en una determinada actividad) y regulación emocional (Schultz, Romanski y Tsatsanis, 2000). Ante estos déficits, lo que se pretende es que vayan edificando sus comportamientos en cuanto a la relación y el proceso interactivo que produce la terapia artística, haciendo hincapié en las necesidades específicas de cada sujeto.

\section{La motivación intrínseca}

La motivación intrínseca es una acción innata del ser humano, de comprometer las capacidades personales e intereses propios y subjetivos para, conseguir un fin, ese algo que se quiere obtener (Deci y Ryan, 1985). Los intereses subjetivos que nos definen y que perseguimos, determinan una verdadera "panacea" de aquello que nos queremos proponer para obtenerlo, conllevando la satisfacción psicológica. Este aspecto es uno de los más importantes que se debe de utilizar para trabajar con él, en estos adolescentes. Según Martín Borreguero (2004), en uno de sus diversos estudios de casos, Luke un sujeto de nueve años, posee dificultades sociales, problemas en su conducta y grandes atisbos de agresividad. Tras una valoración y observación por parte del equipo psicopedagógico del centro donde estudia, exponen lo siguiente: "Luke presenta problemas severos de atención, dificultades de auto regulación de la conducta y una falta de interés y motivación intrínseca por la realización de las tareas administradas".

La Organización Mundial de la Salud (OMS) afirma que las personas con asperger carecen de motivación intrínseca, y esto les afecta en el aprendizaje, ya que esta carestía se debe a la deficiente adaptación de contenidos educativos dirigidos a este colectivo social. ¿Qué conlleva esto? Pues que, quizás, aquellas tareas que han ido dirigidas hacia la educación de Luke, donde el emisor juega un papel muy importante, hubieran tenido unas consecuencias muy diferentes a las que expone la profesora Martín Borreguero si, en este caso, se supiera cuáles son los intereses del niño y encauzar las actividades propuestas, hacia aquellas cosas que despiertan un interés $y$, por lo tanto, una motivación en el niño.

No obstante, se confirma gracias a un programa de intervención en el síndrome de Asperger llevado a cabo en la Universidad de Málaga por Inmaculada Carrasco Juárez y Adrián Guerrero Atencia (2005) donde reafirman que potenciar sus intereses en los contenidos curriculares les ayudará a mostrar una motivación intrínseca hacia aquellas actividades propuestas. 


\section{La creatividad}

La creatividad debe de ser entendida como un proceso en el cual, no solo se van generando ideas desde el interior hacia el exterior, sino también ser sensibles a determinados estímulos externos (aquellos que nos pueden afectar) y dar una solución (o varias) a esos estímulos. Por otro lado, la creatividad también debe de ser entendida como un producto que queremos obtener, es decir, aunque pensamos que lo importante es el resultado final de nuestra creatividad, no es nuestra intención, sino marcar una serie de pautas o procedimientos para conseguir ese producto final.

Según Goñi (2000) la creatividad en el producto es accesible cuando va dirigido a un público en algún momento en concreto, demostrando los intereses, necesidades, etc de los individuos. Sin embargo, las características de una persona con síndrome de Asperger nada tienen que ver con una que consideramos como creativa. Fernández Añino (2003) afirma que según los estudios de Paul Harris, las personas que poseen autismo presentan dificultades a la hora de poder desarrollar un juego creativo o simbólico (también puede ser espontáneo) aunque los adolescentes con asperger, tengan una "rigidez cognitiva", si pueden ser consideradas creativas ante el alto coeficiente intelectual de los individuos reafirmando las tesis de Harris.

A continuación, se exponen algunas razones que determinan que las personas con síndrome de Asperger son creativas según Parada Camelo (2012: 33):
De hecho, algunas de las personalidades más importantes de la historia como Isaac Newton o Albert Einstein tenían síndrome de Asperger, lo que parece demostrar, que no les limitaba en sus acciones creativas. Recientemente, la cantante escocesa Susan Boyle ${ }^{1}$, ganadora de uno de los más famosos concursos de música británica (Britaiin's Got Talent) ha reconocido que tiene síndrome de Asperger (fue diagnosticada hace un año, tiene actualmente 52 años) y en palabras de ella se siente "aliviada" y "relajada".

\section{Las funciones ejecutivas}

Según Delgado y Etchepareborda:

Son habilidades cognitivas propias (...) que permiten establecer metas, diseñar planes, seguir secuencias, seleccionar las conductas apropiadas e iniciar las actividades, así como también autorregular el comportamiento, monitorizar las tareas, seleccionar los comportamientos, y tener flexibilidad en el trabajo cognoscitivo y la organización de la tarea propuesta en el tiempo y en el espacio. (Delgado y Etchepareborda, 2013: 95).

Las capacidades cognitivas básicas sobre las que se fundamentan las funciones ejecutivas son las siguientes:

- Flexibilidad: Establecer la capacidad de elegir entre distintas maneras de actuar, ya que en ocasiones, es necesario cambiar la conducta debido a la acción o tarea que nos requiere.

1.Extraído delartículohttp://www.lavanguardia.com/cultura/20131208/54395297527/ susan-boyle-revela-que-padece-sindrome-de-asperger.html.

\begin{tabular}{ll}
\hline \multicolumn{1}{c}{ Características } & \multicolumn{1}{c}{ Beneficios } \\
\hline -Inteligencia Normal. & $\begin{array}{l}\cdot \text { Como propone Fernández Añino, la inteligencia normal } \\
\text { permitiría al individuo ser una persona creativa. }\end{array}$ \\
Fijación con un determinado tema, llegando a & $\cdot$ Debido a dicha fijación, llegan a dominar todos los aspectos de un \\
ser expertos en el mismo. & $\begin{array}{l}\text { tema. Este amplio conocimiento le permite realizar creaciones } \\
\text { nuevas. }\end{array}$ \\
-Memoria inusual para captar ciertos detalles. & $\begin{array}{l}\cdot \text { Gracias a esta capacidad, son capaces de analizar los pequeños } \\
\text { detalles y descubrir cosas que a otros se les escapan. }\end{array}$ \\
·Personas rutinarias y organizadas. Necesidad & $\begin{array}{l}\cdot \text { Suelen ser muy metódicos, lo que les proporciona una buena } \\
\text { forma de trabajo para investigar. }\end{array}$ \\
\hline
\end{tabular}

Fuente: elaboración propia 
- Planificación: Para lograr un fin o meta, se deberá de establecer un plan de secuencias (rutinas) a nivel sensorial y motor.

- Inhibición: Interrupción de una respuesta automatizada.

- Monitorización: Supervisar los procedimientos o acciones que se están llevando a cabo en la actividad.

- Memoria de trabajo: Absorber cierta información mandada por el emisor, que es necesaria para desarrollarla en un espacio de tiempo en concreto. (Coolidge y Winn, 2001).

Las funciones ejecutivas son necesarias para la mejora de nuevas conductas, basándose en actividades dinámicas y creativas, posibilitando la creación de unas pautas, procedimientos o pasos para llegar a un determinado fin. Por ejemplo, a la hora de establecer una rutina o simplemente que pasos debo dar para poder estudiar un tema de una asignatura concreta (el mapa conceptual es un ejemplo de una de las herramientas más útiles).

Dentro de las funciones ejecutivas es muy importante conocer la flexibilidad cognitiva. Es conceptualizada por el Centro de Investigación en Neurociencias de Rosario (CINR), en la Facultad de Psicología de la Universidad Nacional de Rosario como: La capacidad del sujeto para detectar la ineficacia de sus conductas en ocasiones particulares y la consecuente habilidad para sustituirlas por otras más ajustadas a los requerimientos circunstanciales. La flexibilidad indica capacidad de alternancia cognitiva, opuesta a la rigidez ${ }^{2}$.(Tirapu-Ustárroz, 2002).

En lo que respecta a la evaluación de las funciones ejecutivas, debemos de tener en cuenta la cognición social, es decir, el sustento a través de la teoría de la mente en la cual el sujeto hace referencia a la capacidad cognitiva subjetiva respecto a lo que están sintiendo y pensando en los demás.

2. Concepto escogido del blog en la siguiente dirección http://neurogimn. blogspot.com.es/2008/10/flexibilidad-cognitiva.html.
Es importante el empleo de apoyos visuales con las personas que tienen asperger, ya que destacan por su procesamiento, comprensión y asimilación de la información que están apreciando de manera visual. Por ello, resulta importante trasladar lo visual a cualquier contexto (habilidades de la vida cotidiana, aprendizaje educativo, enseñanza de aptitudes...).

Otros aspectos a destacar, son el favorecimiento de aprendizajes generales, asegurarles un ambiente estable y predecible, evitando cambios que obstaculicen su proceso activo; realizar tareas en pasos más simples, con apenas dificultad, ofrecer distintas posibilidades a la hora de desarrollar una actividad o ayudarles a organizar su tiempo libre evitando la inactividad física y mental o simplemente, limitar su dedicación en cuanto a sus intereses.

\section{Las habilidades sociales}

Las habilidades sociales son las capacidades para comportarse de una forma que es recompensada y en caso de no hacerlo ser castigado o ignorado por los demás (Libet y Lewinsohn, 1973). Sin embargo, otros la definen como la capacidad para interactuar con los demás en un contexto social dado de un modo determinado que es aceptado o valorado socialmente $\mathrm{y}$, al mismo tiempo, personal y mutuamente beneficioso para los demás. (Combs y Slaby, 1977).

Los adolescentes con síndrome de Asperger carecen de habilidades sociales, viéndose limitados a la hora de establecer una conversación recíproca y parecen no entender cuáles son las "reglas" (o herramientas) de la conducta social comunicativa con sus iguales, aprendiéndose a través de la mera observación con los demás.

Por lo tanto, están en una clara desventaja respecto a la sociedad que les rodea. Pero, ¿qué se puede hacer para conseguir esa conducta social comunicativa que tanta falta les hace para desarrollar un acto comunicativo con cualquiera de las personas con la que convive a su alrededor?

Las áreas más importantes para resolver ese "estancamiento" en cuanto a sus habilidades sociales, son las siguientes: 
- Manejar sentimientos y emociones.

- Identificar esos sentimientos y emociones.

- Controlar el estrés.

- Resolver problemas y/o conflictos.

- Habilidades de conversación.

Según Amparo García:

El psicólogo escolar juega un papel crítico, bogando por estos servicios para los alumnos así como también proveyendo instrucción directa a los alumnos y capacitando a los maestros y las familias en varias estrategias, tales como el uso de historias sociales el uso de conversaciones en forma de historietas (Amparo García, 2012: 27)

Para evaluar las habilidades sociales, deberemos fundamentarlas mediante diversos tipos de observaciones y registros. Una entrevista previa con los padres del sujeto a investigar, nos proporcionará información de primera mano acerca de las características y sus circunstancias actuales. También resulta importante, estructurar una entrevista con el docente o psicólogo del sujeto, aportando datos para su funcionamiento con sus iguales.

Además, la utilización de autoinformes en el caso de los adolescentes, dispondremos de la denominada Escala de Habilidades Sociales, propuesta en la página web de la Federación de Asperger de España ${ }^{3}$ donde nos recomienda su utilización. Con el niño podemos utilizar autoinformes. El EHS: Escala de Habilidades Sociales, puede ser también necesario evaluar cuál es el nivel de autoestima del niño. Con frecuencia la falta de recursos sociales lleva anexo la desmotivación, bajo autoconcepto e incluso depresión.

\section{Conclusiones}

El conocimiento del síndrome de Asperger por parte de la sociedad sigue siendo una incógnita por muchos sectores, es de vital importancia darlo a conocer en distintos ámbitos (institutos, colegios, empresas, universidades, medios de comunicación, etc.) partiendo desde el trabajo que se está realizando en asociaciones,

3. www.asperger.es hasta poder lograr una mejor adaptación de este colectivo en la sociedad actual.

Es importante difundir y dar a conocer los distintos y diversos sistemas de comunicación y lenguaje que son utilizados en este Trastorno Generalizado del Desarrollo (TGD), mostrando cuáles son los más aptos para un mayor fomento del aprendizaje del individuo con asperger desde que son niños, para tener una mejor adaptación a la vida adulta.

Existen además, numerosas herramientas audiovisuales que pueden ser de ayuda para el desarrollo social, emocional y comunicativo del individuo con este síndrome. Afrontar las tareas cotidianas ya sea en el colegio, en la asociación de asperger de una ciudad determinada o en el propio hogar, debe de resultar estimulante el aprendizaje de aquellas actividades cotidianas utilizando estas herramientas artísticas y socio-educativas que han sido comentadas a lo largo del texto.

En conclusión, lo visual siempre es más atractivo que lo teórico cuando se está hablando de aprendizaje y enseñanza, por ello, no hay que dejarse engañar por las apariencias y menos, por las capacidades que expresan estos individuos, como bien decía Brassai": "Hay muchas fotografías que están llenas de vida pero son confusas y difíciles de recordar. Es la fuerza de una imagen lo que importa".

\section{Referencias bibliográficas}

Carrasco, I. \& Guerrero, A. (2005). Programa de Intervención en el síndrome de Asperger. Universidad de Málaga. España.

Combs, M. L. \& Slaby, D. L. (1977). Social skills training ith children. En B.B. Lahey y A. E. Kazdin. (Eds): Advances in clinical child psychology. Vol. 1, New York, Plenum.

Coolidge, F. L. y Wynn, T. (2001): Executive functions of the frontal lobes and the evolutionary ascendancy of Homo sapiens. Cambridge Archaeol. Journal 11: 255-260.

4. De nacionalidad húngara, este fotógrafo y profesor de literatura, desarrolló la mayor parte de su vida académica en París siendo uno de los fotógrafos de Hungría mejor conocidos. 
Deci, E. L., \& Ryan, R. M. (1985). Intrinsic motivation and self-determination in human behavior. New York: Plenum.

Delgado, I. \& Etchepareborda, M. (2013). Trastornos de las funciones ejecutivas. Diagnóstico y tratamiento. Revista Neurología, 57 (Supl 1).pp. $95-103$.

Duarte Santos, G. \& Simoes, M. (2011). Mediadores artístico-expressivos e Interacção Social - Estudo com crianças com Perturbação do Espectro do Autismo. International Journal of Developmental and Educational Psychology, XXIII, No 1 (1).

Eisner, E. W. (2004). El arte y la creación de la mente: el papel de las artes visuales en la transformación de la conciencia. Barcelona: Ed. Paidós.

Elkis-Albuhoff, D. (2008). Art Therapy applied to an adolescent with Asperger's syndrome. The arts in Psycotherapy, 35, 262-270.

Emery, M. J. (2004). Arte Therapy as an intervention for Autism. Art Therapy: Journal of the American Art Therapy Association, 21, (3), 143-147.

Epp, K. M. (2008). Outcome-based Evaluation of a social skills Program using Art Therapy and Group Therapy for children on the autism spectrum. Children and Schools, 30, (1), $27-36$.

Evans, K., \& Dubowski, J. (2001). Art therapy with children on the autistic spectrum disorder. London, UK: Jessica Kingsley.

Federación Asperger España. Tomado de: http:// www.asperger.es/asperger.php?t=3.

Fernández Añino, $\mathbf{M}^{\mathrm{a}}$. (2003). Creatividad, arte terapia y autismo. Un acercamiento a la actividad Plástica como proceso creativo en niños autistas. Revista Arte, individuo y sociedad, 15, 135-152.
Gabriels, R. L. (2000). Art therapy with children who have autism and their families. En C. A. Malchiodi (Ed.), Handbook of art therapy (pp. 193-206). New York: Guildford.

García, A. (2012). Habilidades sociales: una aplicación al síndrome de Asperger. Practicum de la Facultad de Psicología. Universidad Oberta de Catalunya.

Gray C. (1994). Mi libro de historias sociales. (Recuperado el 14/12/13). https://skydrive. live.com/?cid $=$ cc39baa414d088bd\&id $=\mathrm{CC}$ 39BAA414D088BD\%21177.

Goñi, A. (2000). Desarrollo de la creatividad. San José: Euned.

Henley, D. (2000). Blessings in disguise: Idiomatic expression as a stimulus in group art therapy with children. Art Therapy: Journal of the American Art Therapy Association, 17, 270-275.

Klein, J.P, Bassols, M. y Bonet, E. (Coord.) (2008). Arteterapia. La creación como proceso de transformación. Barcelona, Octaedro.

Klein, J.P. (2006). Arteterapia. Una introducción. Barcelona, Octaedro.

Libet, J \& \& Lewinsohn, M. (1973). The concept of social ski with special reference to the behavior of depressed persons. Journal of Consulting and Clinical Psychology 40, pp. 304-312.

Martín Borreguero, P. (2004). El síndrome de Asperger: ¿Excentricidad o discapacidad social? Madrid: Alianza Editorial.

Parada, A. (2012). El síndrome de Asperger y la creatividad: el desarrollo de las habilidades sociales a través del Arte-Terapia. Universidad de Oviedo.

Rosal, M. L. (1996). Approaches to art therapy with children. Burlingame, C.A, Abbeygate. 
Rosal, M. L. (2001). Cognitive-Behavioral Art Therapy. En Rubin J. A. (Eds). Approaches to Art Therapy: Theory and Thecnique. pp. 210 - 225. Nueva York, Brunner-Routledge.

Schultz, T. T., Romanski, L. M., \& Tsatsanis, K. D. (2000). Neurofunctional models of Asperger Syndrome. En A. Klin, F. R. Volkmar, \& S. S. Sparrow (Eds.), Asperger syndrome (pp. 340-366). New York: Guildford. 